\title{
Chest ultrasound in diagnosis of pneumonia in children: A descriptive cross-sectional hospital based study
}

Ali Abo Elmagd Ahmed 1, Ashraf Mahmoud Radwan 2, Mohammed Alzaky Ali 3,Ebtesam Mohammed Mohammed 4.

1professor of Pediatric Department,Faculty of Medicine, Sohag University, Sohag, Egypt. 2 Lecturer of Pediatric Department, Faculty of Medicine,Sohag University, Sohag, Egypt.

3 Lecturer of RadiologyDepartment, Faculty of Medicine, Sohag University, Sohag, Egypt.

4 Specialist, Pediatric Department,Faculty of Medicine, Sohag University, Sohag Egypt.

Type of article: Original

\section{Abstract}

Background:Pneumonia is defined as "new lung infiltrates plus clinical evidence that the infiltrate is of an infectious origin, which include the new onset of fever, purulent sputum, leukocytosis, and decline in oxygenation. LUS has a number of advantages it is easy to do at a child's bedside, takes little time to perform and interpret the results, allows a close follow-up, and avoids the use of ionising radiation.

Objective: Our main objective in this study is to validate the value of lung ultrasound in diagnosis of pneumonia in children in comparison with chest radiography in Sohag University Hospital.

Methods: This adescriptive cross-sectional study of children clinically diagnosed as pneumonia conducted on 70 cases they were classified into four groups according to the age of patients; group I from < 1 month to 1 month, group II from 1 month to 12 month group III from 1 year to 4 years, group IV from 4 to 11 years over period of six months fromAugustus 2015toJanuary 2016 in Pediatric department, neonatal care unit in Sohag University Hospital.The data was analyzed by SPSS software.

Results: LUS findings among studied population, consolidation was seen in 66 cases of 70 (94.29\%),30 cases of them were in the right lung, 22 were in the left one $(33.33 \%)$ and 14cases were bilateral (21.21\%.(pleural effusion was detected in 28 cases $(40 \%)$ and cannot be detected in 42 patients (60\%).comparison between LUS findings and diagnosis of pneumonia ,there was no statistical significant difference between LUS findings and diagnosing pneumonia this indicated that LUS is helpful and accurate in diagnosis of pneumonia in children .

Conclusion: chest ultrasound was accurate in the diagnosis of pneumonia and we suggest that LUS is an alternative tool to chest radiography in the diagnosis of pneumonia in children and that the follow up of patients with pneumonia by LUS can reduce the exposure of children to ionizing radiation.

Key words: diagnosis of pneumonia in children and Chest ultrasound.

\section{Introduction}

Pneumonia is defined as "new lung infiltrates plus clinical evidence that the infiltrate is of an infectious origin, which include the new onset of fever, purulent sputum, leukocytosis, and decline in oxygenation (Cunha BA, 2010).

Pediatric pneumonia is the number one cause of death by illness in children worldwide. It can be especially difficult to diagnose because symptoms vary according to achild's age and cause of infection (Lomas DJ et al, 2001).

The current guidelines suggest that the diagnosis of pneumonia can only be made on the clinical history, respiratory rate, fever, respiratory signs and symptoms reserving the use of radiography only in severe or complicated cases (Harris et al., 2011; Bradley et al., 2011). 
Despite these latest indications chest radiography is commonly considered the best choice for the diagnosis of pneumonia among physicians and its execution is also requested for mild cases because of the poor reliability of the history and physical examination (Shah et al., 2010; Ayalon et al., 2013).

\section{Material and methods:}

This a descriptive cross-sectional study of children clinically diagnosed as pneumonia conducted on 70 cases they were classified into four groups according to the age of patients; group I from <1 month to 1month, group II from 1 month to 12 month group III from 1 year to 4 years, group IV from 4 to 11 years over period of six months from Augustus 2015 to January 2016 in Pediatric department, neonatal care unit in Sohag University Hospital. The patients were initially interviewed, clinically examined, and then underwent chest ultrasound. Those participants who have congenital heart disease, rheumatic heart disease and other known cardiac diseases were excluded from the study. Written consent was obtained from each participant and the study was approved by the medical ethics committee of
Sohag University of Medical Science. Those patients underwent complete general andRoutine laboratory tests: arterial blood gases, complete blood count, Liver and kidney functions tests, C-reactive protein and erythrocyte sedimentation rate (ESR) chest examination and Chest ultrasound: performed within $24 \mathrm{~h}$ after the chest $\mathrm{x}$ ray. Sonography was assessed for the location, shape, size and echogenicity of consolidation as well as necrotic areas, positive air bronchograme, and pleural effusion if present.

\section{Statistical analysis:}

Data was analyzed using STATA intercooled version 12.1. Qualitative data was presented as number and percentage and compared using either Chi square test or fisher exact test. The concordance of US and CR in identifying consolidation and pleural effusion was evaluated using Cohen's weighted kappa (k) statistics. The discrepancies between US and CR were assessed using marginal homogeneity Stuart-Maxwell statistics (the McNemar chi-squared test for paired data give the same results). Graphs were produced by using Excel program. $P$ value was considered significant if it was less than 0.05 .

\section{Results:}

Table (1) showed age groups from below 1 month up to 11years, Group I: from <1 month up to 1 month, only 10 cases of about $14.29 \%$, Group II: from 1month up to 12 month, was 16 cases of about $22.86 \%$, Group III : from 1 year up to 4 years, it was 22 cases of about $31.43 \%$, Group IV: from 4 years up to 11 years, it was 22 cases of about $31.43 \%$, (equal to Group III ( Also it showed sex distribution of 70 cases 36 of them were females $(51 \%)$ and 34 were males (49\%) with female predominance.Table (2) showed the findings of LUS findings among studied population, consolidation was seen in 66 cases of $70(94.29 \%), 30$ cases of them were in the right lung, 22 were in the left one $(33.33 \%)$ and 14 cases were bilateral $(21.21 \%)$, pleural effusion was detected in 28 cases $(40 \%)$ and cannot be detected in 42 patients $(60 \%)$. Table (3) showed comparison between LUS findings 
and diagnosis of pneumonia, there was no statistical significant difference between LUS findings and diagnosing pneumonia this indicated that LUS is helpful and accurate in diagnosis of pneumonia in children.

Table (1): Age and sex distribution of studied population:

\begin{tabular}{|l|l|}
\hline Variable & Summary statistics \\
\hline Age group & \\
$<1$ month & $10(14.29 \%)$ \\
$1-12$ month & $16(22.86 \%)$ \\
$1-4$ year & $22(31.43 \%)$ \\
$5-11$ years & $22(31.43 \%)$ \\
\hline Sex group & $36(51.43 \%)$ \\
Females & $34(48.57 \%)$ \\
Males & \\
\hline
\end{tabular}

Table (2): lung Ultrasonographic findings among studied population:

\begin{tabular}{|l|l|}
\hline Variable & Summary statistics \\
\hline $\begin{array}{l}\text { Consolidation } \\
\text { Negative } \\
\text { Positive }\end{array}$ & $4(5.71 \%)$ \\
\hline Site of consolidation & $66(94.29 \%)$ \\
Left & $22(33.33 \%)$ \\
Right & $30(45.45 \%)$ \\
Bilateral & $14(21.21 \%)$ \\
\hline Pleural effusion & \\
No & $42(60.00 \%)$ \\
Yes & $28(40.00 \%)$ \\
\hline
\end{tabular}

Table (3): Comparison between Ultrasonography finding and diagnosis of pneumonia as regard consolidation.

\begin{tabular}{|l|l|l|l|}
\hline & Ultrasonography finding & Diagnosis of pneumonia & P value \\
\hline No & $4(5.711 \%)$ & $\mathbf{0}$ & 0.12 \\
Yes & $66(94.29 \%)$ & $\mathbf{7 0}(\mathbf{1 0 0 \% )})$ & \\
\hline
\end{tabular}

\section{Discussion:}

In our study there were 70 cases they were classified into four groups according to the age of patients ; group I from <1 month to 1month, group II from 1 month to 12 month group III from 1 year to 4 years, group IV from 4 to 11 years. This age distribution was used in the study done in Italy in 2015, whereas selected cases were ranged in the same matter from age of 2 months to 12 years (preda et al,2015), also was similar to that study in Italy in 2014 whereas the age distribution was from 0 to $<16$ years (Reali et al,2014). The sex distribution of our study showed female predominance as females were 36 out of 70 cases $(51 \%)$ while males were 34 cases $(49 \%)$, this distribution was similar to the study done in Italy in 2008 whereas males 
were 37 out of 79 cases $(46.8 \%)$ and females were 42 cases $(53.1 \%)$, also it was similar to that study done in Egypt in 2013 in Cairo University Pediatric Hospital whereas males were 36 cases out of 75 cases (48\%) and females were 39 cases $(52 \%)$, (Seif El Din et al,2013). .In our study by means of LUS consolidation was seen in 66 patients out of 70 (94.29\%).This result was more or less similar to that done in Tiwan wheres 159 patients out of 163 (97.2\%) showed consolidation,(MengChiehHo.,et al2014).Also more or less near the result done in Italy in 2015 on 29 cases confirmed as pneumonia 28 of them showed consolidation $(96.5 \%)$, ( Iorio.,et al 2015) and also similar to the result of study done in Italy on 81 cases confirmed as pneumonia 76 of them were positive for consolidation (94\%), (Reali.,et al 2014).LUS agreed in diagnosis of pneumonia in 48 cases out of $70(68.57 \%)$ and 4 cases $(5.7 \%)$ diagnosed by CXR can't be seen by LUS with no statistical significant difference $(p$ value $=0.12)$. This issue was addressed by shah et al in the study done in USA in 2013 (shah et al., 2013).

Conclusions:our study showed a high detection rate for LUS in identifying pneumonia in children. It also may be a promising tool for the follow up of patients with pneumonia.Patients can receive more frequent follow ups using LUS during treatment and more information can be made available to pediatricians for decision-making.

Acknowledgment:Authors thank colleagues in the Pediatric Department, Faculty of Medicine, Sohag University (Egypt) for their assistance, and the parents and children who participated in this study.

Conflict of Interest:There is no conflict of interest to be declared.

Authorscontributions:All authors contributed to this project and article equally. All authors read and approved the final manuscript.

\section{References:}

1. Ayalon I, Glatstein MM, ZaidenbergIsraeli G, Scolnik D, Ben Tov A, Ben Sira L, Reif S. The role of physical examination in establishing the diagnosis of pneumonia. Pediatric Emergency Care. 2013;29(8):893-896. doi:.1097/PEC.0b013e31829e7d6a.

2. Bradley JS, Byington CL, Shah SS, Alverson B, Carter ER, Harrison C, Kaplan SL, Mace SE, McCracken GH, Jr, Moore MR, St Peter SD, Stockwell JA, Swanson JT. The management of community-acquired pneumonia in infants and children older than 3 months of age: clinical practice guidelines by the Pediatric Infectious Diseases Society and the Infectious Diseases Society of America. Clinical Infectious Diseases. 2011;53:e1374doi: 10.1093/cid/cir531.

3. Cunha BA. Swine Influenza (H1N1) Pneumonia: Clinical Considerations. Infect Dis Clin N Am. 2010. 24:203228.

4. GiulioIorio, Maria Capasso,Giuseppe De Luca, Salvatore Prisco,CarloMancusi, Bruno Laganà,and Vincenzo Comune1PeerJ. 2015; 3: e1374.Published online 2015 Nov 10. doi: 10.7717/peerj.1374.

5. Lomas DJ, Padley SG, Flower CD. The sonographic appearances of pleural fluid. Br J Radiol 1993; 66:619-624. Abstract/FREE Full Text Ghaye B, Dondelinger RF. Imaging guided thoracic interventions. EurRespir J 2001; 17:507-528.

6. Meng-Chieh Ho, Chin-Ru Ker a, JongHau Hsu b,c,dJiunn-Ren Wu b,c,d, Ze006E,Kong Dai b,c,d,y, I-Chen Chen, chest radiography children;lungultrasound;pneumonia, Pediatrics, 2014 http://dx.doi.org/10.1016/j.pedneo.2014. 03.007 .

7. Pereda MA, Chavez MA, Hooper-Miele CC, Gilman RH, Steinhoff MC, 
Ellington LE, Gross M, Price C, Tielsch JM, Checkley W. Lung ultrasound for the diagnosis of pneumonia in children: a meta-analysis. Pediatrics. 2015;135(4):714-722. doi: 10.1542/peds.2014-2833.

8. Reali F, Sferrazza Papa GF, Carlucci P, Fracasso P, Di Marco F, Mandelli M, Soldi S, Riva E, Centanni S. Can lung ultrasound replace chest radiography for the diagnosis of pneumonia in hospitalized children? Respiration. 2014; 88:112-115. doi: $0.1159 / 000362692$.

9. Seif El Dien HM, Abd Ellatif DAK. The value of bedside lung ultrasonography in diagnosis of neonatal pneumonia. Egypt J RadiolNuc Med. 2013;44(2):339-347.

10. Shah S, Bachur R, Kim D, Neuman MI. Lack of predictive value of tachypnea in thediagnosis of pneumonia in children. Pediatric Infectious Disease Journal. 2010 ; 29 (5) : 406 - 409. Doi : 10. 1097/ INF. 0b013e3181cb45a7.

11.Shah, Tunik\&Tsung (2013) Shah VP, Tunik MG, Tsung JW. Prospective evaluation of point-of-care ultrasonography for the diagnosis of pneumonia in children and young adults. JAMA Pediatrics. 2013; 167:119-125. doi: 10.1001/2013.jamapediatrics.107. 